\title{
PRUEBAS DE GERMINACIÓN DE SEMILLAS DE FORESTALES NATIVOS DE CORDOBA, COLOMBIA, EN LABORATORIO Y CASA-MALLA
}

\section{SEED GERMINATION TESTS OF NATIVE FOREST SPECIES OF CORDOBA, COLOMBIA IN LABORATORY AND GREENHOUSE}

\author{
Miguel Espitia ${ }^{1}$, Carlos Cardona ${ }^{2}$, Hermes Araméndiz $^{3}$ \\ ${ }^{1}$ I.A. Ph.D. Facultad de Ciencias Agrícolas. Universidad de Córdoba, Montería, Colombia, Diagonal 50 No.8-105, Montería, \\ Córdoba, Colombia, e-mail: mmespitia@correo.unicordoba.edu.co; ${ }^{2}$ I.A. Ph.D., Facultad de Ciencias Agrícolas. Universidad \\ de Córdoba, Montería, Colombia, e-mail: ccardonaayala@yahoo.com; ${ }^{3}$ I.A. Ph.D. Facultad de Ciencias Agrícolas. Universidad \\ de Córdoba, Montería, Colombia, e-mail: haramendiz@hotmail.com
}

Rev. U.D.C.A Act. \& Div.Cient. 19(2): 307-315, Julio-Diciembre, 2016

\section{RESUMEN}

El objetivo del estudio fue evaluar la germinación de las semillas de cinco especies forestales nativas de Córdoba: Cedrela odorata, Cariniana pyriformis, Bombacopsis quinata, Anacardium excelsum y Schizolobium parahybum. El estudio, se realizó entre abril de 2013 y mayo de 2014, en el Laboratorio de fitomejoramiento de la Universidad de Córdoba. En cada uno de los dos ambientes de germinación: cámara germinativa y casamalla, se utilizó un diseño completamente al azar, con cinco especies forestales como tratamientos y cuatro repeticiones de 50 semillas cada una. Toda la semilla fue sumergida en agua destilada, a temperatura ambiente de $27^{\circ} \mathrm{C}$, por 24 horas, antes de la siembra. Se estimaron y compararon las variables, porcentaje de germinación (PG), índice de velocidad de germinación (IVG), germinación diaria media (GDM), valor pico (VP) y valor de germinación (VG). Los resultados mostraron diferencias estadísticas entre especies e interacción especies por ambientes de germinación en el análisis combinado de varianza, para los parámetros fisiológicos de la germinación evaluados, a excepción del PG. Los valores de los parámetros de germinación fueron mayores en cámara de germinación, con incrementos entre el 48,2 y $124,2 \%$, en GDM y VG, respectivamente. $A$. excelsum mostró las mayores diferencias entre especies en los dos ambientes de germinación en todos los parámetros germinativos, mientras que $B$. quinata presentó las menores diferencias. Se recomienda estimar los parámetros de germinación en cámara germinativa, en lugar de casa-malla, siempre que se disponga de este equipo.

\section{SUMMARY}

The objective of this study was to evaluate the germination of seeds of five native forest species of Córdoba: Cedrela odorota, Cariniana pyriformis, Bombacopsis quinata, Anacardium excelsum and Schizolobium parahybum. The study was conducted between April of 2013 and May of 2014 at the Laboratory of Plant Breeding at the Universidad de Córdoba. A completely randomized design was used in each environment: germination chamber and greenhouse with five forest species as treatments and four replicates of 50 seeds each. All seeds were immersed in distilled water at room temperature of $27^{\circ} \mathrm{C}$ for 24 hours before planting. The parameters germination percentage (GP), germination speed index (GSI), mean daily germination (MDG), peak value (PV) and germination value (GV) were estimated and compared. The results showed statistical differences between species and interaction species $\mathrm{x}$ germination environments in combined analysis of variance for germination physiological parameters evaluated, except for PG. The values of germination parameter estimated were higher in germination chamber, with increases between $48.2 \%$ and $124.2 \%$ in MDG and GV, respectively. A. excelsum showed the greatest differences between species in two germination environments in all parameters estimated, while $B$. quinata showed minor differences. It is recommended to estimate the parameters in germination chambers, instead of greenhouse, whenever this equipment is available.

Key words: Trees species, germination chamber, germination parameters.

Palabras clave: Especies arbóreas, cámara germinativa, parámetros germinativos. 


\section{INTRODUCCIÓN}

En el 2014, el departamento de Córdoba registró un área forestal plantada de 32.800ha, de las cuales, 11.800ha fueron sembradas con diferentes especies forestales nativas, en las que predominan Tabebuia rosea (roble) y Bombacopsis. quinata (ceiba). Las especies en estudio tienen su importancia, en general, por su gran adaptación a las variaciones edafoclimáticas de la región, aportes de servicios ecosistémicos, buen conocimiento por reforestadores y procesadores de la madera, variado uso en productos maderables y no maderables, excelente calidad de la madera y materia prima para uso en dendroenergía, fabricación de viviendas, artesanías y muebles finos, entre otros (Campo-Arana et al. 2014).

La mayoría de las especies forestales del trópico, se propagan mediante semilla sexual y su calidad fisiológica, genética, física y sanitaria influye, de manera significativa, en el éxito de la producción y productividad de las plantaciones. Las empresas reforestadoras, viveristas y bancos de germoplasma requieren información segura, confiable y de calidad sobre manejo en vivero y conservación de las semillas de especies forestales (Niembro \& Ramírez-García, 2006; Nascimento, 2013).

El conocimiento e interpretación de la viabilidad y los parámetros de la germinación y las plántulas en laboratorio, invernadero o casa-malla es indispensable para la comprensión de la autoecología, colecta, conservación, manejo, uso sostenible y el desarrollo de prácticas silviculturales exitosas de las especies (Abbade \& Massanori, 2014; Deminicis et al. 2014; Guimarães et al. 2015).

Varias investigaciones han reportado diferencias estadísticas en los parámetros de calidad fisiológica de las semillas en diferentes especies, lotes familiares de una misma especie o diferentes procedencias en varias especies forestales o arbustivas (Silva et al. 2012; Ferreira et al. 2012; Correa et al. 2013; Zohra et al. 2014; Barboza-Nogueira et al. 2014). Adicionalmente, otros estudios en Cotinus coggygria (Olmez et al. 2009), Punica granatum (Gokturk et al. 2012), Bromus tomentellus, Bromus inermis y Bromus persicus (Armaki et al. 2013) y en Poa bulbosa, Poa annua y Poa pratensis (Armaki, 2014) han detectado diferencias estadísticas en los parámetros de la germinación de la semillas, a nivel de especies y con valores más altos en las condiciones de cámaras germinativas, en comparación con las obtenidas en invernaderos.

En Córdoba, las especies forestales nativas de interés han sido poco investigadas en su respuesta y diferencia en los parámetros fisiológicos de la germinación de las semillas, cuando las pruebas se realizan en cámara germinativa comparadas con las mismas en casa-malla. Estos estudios, se han reconocido como de fundamental importancia para el uso sostenible, conservación, multiplicación, reforestación y mejoramiento genético de tales especies promisorias de Córdoba y Colombia (Correa et al. 2013).

El presente estudio tuvo como objetivo principal comparar cinco parámetros fisiológicos de la germinación de las semillas en condiciones de cámara germinativa y casamalla, para cinco especies forestales nativas priorizadas, en el departamento de Córdoba: Cedrela odorata L, (Cedro), Cariniana pyriformis Miers (Abarco), Bombacopsis quinata (Jacq.) Dugand (Ceiba roja), Anacardium excelsum (Bert. et Balb) Skeels (Caracolí) y Schizolobium parahybum (Vell.) Blake (Tambor).

\section{MATERIALES Y MÉTODOS}

El estudio, se adelantó entre abril de 2013 y julio de 2014, en la casa-malla y la cámara germinativa del laboratorio de fitomejoramiento de la Universidad de Córdoba, ubicada en la zona media del valle del Sinú, a $8^{\circ} 52^{\prime}$ de latitud Norte y $76^{\circ} 48^{\prime}$ longitud Oeste, a una altura de $13 \mathrm{msnm}$. La zona ecológica corresponde a bosque seco tropical, con temperatura promedio de $28^{\circ} \mathrm{C}$, humedad relativa de $84 \%$ y precipitación anual de $1.200 \mathrm{~mm}$ (Palencia et al. 2006).

Se evaluó un compuesto balanceado de 15 lotes familiares de semilla sexual de libre polinización de sendos árboles, seleccionados en seis zonas diferentes del departamento de Córdoba, en cada una de las cinco especies: $C$. odorata, $C$. pyriformis, B. quinata, A. excelsum y S. parahybum.

Las zonas forestales, donde se realizó la colecta de la semilla, presentan diferencias agroclimáticas, especialmente, en la precipitación que varía desde 1.231 hasta $1.760 \mathrm{~mm} /$ año. La toma de las muestras de semillas, se realizó con base en los protocolos específicos propuestos por Gold et al. (2004). Las áreas forestales muestreadas, se ubicaron en las ciudades de Montería, San Carlos, San Antero, Planeta Rica, Tierralta y Ciénaga de Oro, del departamento de Córdoba.

Para el estudio, se estimaron cinco variables de respuesta relacionadas con los parámetros fisiológicos de la germinación de las semillas en sendas pruebas de germinación en cámara germinativa (CAGER) y casa-malla (CAMA), para cada una de las cinco especies forestales en estudio. Los parámetros de la germinación estimados fueron: porcentaje de germinación acumulado (PG), índice de velocidad de germinación (IVG), germinación diaria media (GDM), valor pico (VP) y valor de germinación (VG).

En cada especie, se realizó un pre-acondicionamiento de las semillas, sumergiéndolas en agua destilada, a temperatura 
ambiente de $27^{\circ} \mathrm{C}$. En las especies C. odorata, C. pyriformis, $B$. quinata y $A$. excelsum, se utilizó un tiempo de inmersión de $24 \mathrm{~h}$, lapso que está dentro de los rangos aceptados para las especies arbóreas (Correa et al. 2013). En el caso de las semillas de $S$. parahybum, las cuales, presentan una cubierta muy dura, fueron sumergidas en agua destilada durante 48 horas, a temperatura ambiente.

Las pruebas de germinación en CAGER fueron realizadas en bandejas de aluminio, con sustrato de cuatro capas de papel toalla blanco húmedo, encima del cual, se colocaron las semillas y luego, se cubrieron con otra cantidad igual de papel toalla húmeda. Las bandejas con las semillas fueron colocadas, posteriormente, dentro de una cámara de germinación, marca Dies ${ }^{\circledR}$, a una temperatura de $28^{\circ} \mathrm{C}$, humedad relativa de $80 \%$, con periodo de luz de $10 \mathrm{~h} /$ día y regadas diariamente de forma uniforme. Las pruebas de germinación, en condiciones de CAMA, fueron implementadas con recubrimiento de polipropileno color negro, malla antiáfidos, temperatura media de $29^{\circ} \mathrm{C}$, humedad relativa del $70 \%$ y sombreamiento de $33 \%$. Las semillas fueron sembradas a una distancia de $5 \mathrm{~cm}$ entre hileras y $3 \mathrm{~cm}$ entre semillas, en bandejas de aluminio, con un sustrato uniforme, conformado de $50 \%$ de arena cuarcítica y $50 \%$ de arcilla, ambos desinfectados con agua caliente. La profundidad de siembra fue de 2/3 del tamaño de la semilla, con la parte por donde emerge la radícula hacia abajo. Se realizaron dos riegos diarios a las 10a.m. y a las 4p.m.; la germinación fue valorada diariamente durante 55 días. Se consideró la germinación cuando los cotiledones se levantaron del nivel del sustrato.

El PG fue considerado como el porcentaje acumulado de semillas germinadas al final del ensayo; el IVG fue calculado mediante la fórmula recomendada por Maguire (1962):

$\mathrm{IVG}=\frac{p_{1}}{T 1}+\frac{P_{2}}{T 2}+\frac{p_{3}}{T 3}+\cdots \frac{p_{n}}{T n}$

Donde: $\mathrm{P}_{1}, \mathrm{P}_{2}, \mathrm{P}_{3} \ldots, \mathrm{Pn}=$ número de plántulas normales, germinadas y completas en el primer, segundo, tercer y último conteo de la evaluación.

$T_{1}, T_{2}, T_{3}, \ldots, T_{n}=$ tiempo en días para cada germinación.

La GDM, se consideró como la relación entre el porcentaje acumulado de semillas germinadas al final del ensayo y el número de días desde la siembra al término del ensayo; el VP como la GDM máxima alcanzada en el ensayo y el VG, correspondió al producto de la GDM por el VP (Czabator, 1962).

En cada uno de los dos ambientes de germinación: CAGER y CAMA, se utilizó un diseño completamente al azar, con cinco especies forestales como tratamientos y cuatro repeticiones de 50 semillas cada una. Una vez establecida la homogeneidad de varianzas de los errores de los dos ensayos, se procedió a efectuar el análisis combinado de varianza y las pruebas de comparación de medias de Duncan. Los análisis estadísticos, se realizaron mediante el uso del programa computacional GENES versión Windows (2009.7.0), desarrollado por Cruz (2014).

\section{RESULTADOS Y DISCUSIÓN}

Análisis combinado de varianza (ANACOMVA). Se detectaron diferencias estadísticas significativas $(P<0,01)$ entre especies (E) e interacción especies por ambientes germinativos (ExA) en el análisis combinado de varianza (Tabla 1), para los parámetros fisiológicos de la germinación de las semillas evaluados, a excepción del porcentaje de germinación (PG), lo que indica que, al menos, una de las especies en estudio presentó promedio estadísticamente diferente y su respuesta cambió en los dos ambientes germinativos para el índice de velocidad de germinación (IVG), germinación diaria media (GDM), valor pico (VP) y valor de germinación (VG). Resultados similares han sido reportados en lotes familiares o procedencias de semillas en especies forestales o arbustivas, como Swietenia macrophylla, de acuerdo con Niembro \& Ramírez-García (2006); cinco especies maderables del Tibet, reportadas por Wang et al. (2010); Bauhinia thonningii, conforme a Mwase \& Mvula (2011); Quercus rugosa, de acuerdo a Huerta-Paniagua \& Rodríguez-Trejo (2011); Pachira aquatica, según Silva et al. (2012); Triplaris surinamensis, reportada por Ferreira et al. (2012); Tectona grandis, conforme a Correa et al. (2013); Argania spinosa, de acuerdo con Zohra et al. (2014); Anadenanthera colubrina, según Barboza-Nogueira et al. (2014). Este resultado evidencia las diferencias genéticas y el comportamiento diferencial existentes entre las semillas de las especies consideradas en los parámetros de la germinación, que resultaron estadísticamente significativos.

Porcentaje de germinación acumulado (PG). En la tabla 2 , se observa que la media general para el PG entre las especies, osciló entre $70,5 \%$, para C. pyriformis y $77,5 \%$, para C. odorata, con una media general para todo el estudio de $73,9 \%$, sin existir diferencias estadísticas entre las especies. Los promedios en PG, de 76,8 y 71,0\%, para cámara germinativa (CAGER) y casa-malla (CAMA), respectivamente, corroboran lo obtenido en el ANACOMVA (Tabla 1). Estos resultados sugieren que las semillas de las cinco especies germinaron de manera similar, a nivel general y en cada ambiente germinativo; no obstante, se observa que en CAGER germinó un 8,2\% de mayor número de semillas que en CAMA. Esta tendencia, se expresa con mayor importancia en $A$. excelsum, con incrementos estadísticamente significativos, del 22,7\%, a favor CAGER. Ello se explica por el mejor control de las condiciones 
Tabla 1. Análisis combinado de varianza y nivel de significancia para cinco parámetros fisiológicos de la germinación bajo dos ambientes germinativos de las semillas, para cinco especies forestales nativas en Córdoba.

\begin{tabular}{|l|c|r|r|r|r|r|}
\hline \multirow{2}{*}{ F.V. } & \multirow{2}{*}{ g.l. } & \multicolumn{5}{|c|}{ CM } \\
\cline { 3 - 7 } & & PG (\%) & IVG (ptas/dia) & GDM (\%) & VP (\%) & \multicolumn{1}{c|}{ VG (\%) } \\
\hline Ambientes (A) & 1 & $336,40 \mathrm{~ns}$ & $27,42 \mathrm{~ns}$ & $28,97 \mathrm{~ns}$ & $207,53 \mathrm{~ns}$ & $14345,16 \mathrm{~ns}$ \\
\hline Especies (E) & 4 & $49,40 \mathrm{~ns}$ & $26,68^{* *}$ & $44,19 * *$ & $214,00^{* *}$ & $16927,25^{* *}$ \\
\hline ExA & 4 & $77,40 \mathrm{~ns}$ & $8,07 * *$ & $11,52^{* *}$ & $65,89 * *$ & $6793,70^{* *}$ \\
\hline Error Comb. & 30 & 67,87 & 0,25 & 0,27 & 2,14 & 252,42 \\
\hline Total & 39 & & & & & \\
\hline Media & & 73,90 & 3,00 & 4,38 & 8,06 & 49,43 \\
\hline CV(\%) & & 11,15 & 16,50 & 11,75 & 18,17 & 32,14 \\
\hline
\end{tabular}

CM: Cuadrados medios del análisis combinado de varianza. ns: no significativos y ** significativos al $1 \%$ de probabilidad, respectivamente. PG: Porcentaje de germinación acumulado; IVG: Índice de velocidad germinativo; GDM: Germinación diaria media; VP: Valor pico y VG: Valor germinativo. Ambientes: ambientes germinativos: cámara germinativa y casa-malla. Error Comb.: error combinado.

ambientales para la germinación en CAGER, principalmente, temperatura y humedad.

Índice de velocidad de la germinación (IVG). Los valores promedios (Tabla 2) corroboran los resultados obtenidos en el ANACOMVA, a nivel de ambientes (A), especies (E) e interacción ExA. A pesar que cuando se presenta una interacción ExA estadísticamente significativa, se deben analizar los resultados por separado en cada ambiente germinativo; vale la pena resaltar que, en general, $C$. pyriformis y S. parahybum registraron los valores más bajos de IVG, con 1,13 y 1,57 plántulas/día, en su orden, mientras que $B$. quinata registró el mayor valor promedio de IVG, con 5,71 plántulas/día; este valor fue superior a la media general en 2,71 plántulas/día y destaca $B$. quinata, como una especie con alta energía germinativa, en razón a que, de acuerdo con Maguire (1962), los mayores valores de IVG están relacionados con mayor energía para germinar de las semillas, mientras que $C$. pyriformis y $S$. parahybum se caracterizan por su baja energía germinativa.

Tabla 2. Promedios de porcentaje acumulado de germinación (PG), índice de velocidad de la germinación (IVG) y germinación diaria media (GDM) de las semillas en cámara germinativa (CAGER) y casa-malla (CAMA), para cinco especies forestales nativas en Córdoba.

\begin{tabular}{|l|c|c|c|c|c|c|c|c|c|c|c|c|}
\hline \multirow{2}{*}{ Especies } & \multicolumn{4}{|c|}{ PG (\%) } & \multicolumn{4}{c|}{ IVG (ptas/dia) } & \multicolumn{4}{c|}{ GDM (\%) } \\
\cline { 2 - 16 } & Media & CAGER & CAMA & INC (\%) & Media & CAGER & CAMA & INC (\%) & Media & CAGER & CAMA & INC (\%) \\
\hline C. odorata & $77,5 \mathrm{a}$ & $82 \mathrm{a}$ & $73 \mathrm{ab}$ & 12,3 & $3,67 \mathrm{~b}$ & $5,72 \mathrm{a}$ & $1,63 \mathrm{~cd}$ & 250,8 & $6,70 \mathrm{a}$ & $9,11 \mathrm{a}$ & $4,29 \mathrm{~d}$ & 112,2 \\
\hline S. parahybum & $74,0 \mathrm{a}$ & $76 \mathrm{ab}$ & $72 \mathrm{ab}$ & 5,6 & $1,57 \mathrm{~d}$ & $1,69 \mathrm{c}$ & $1,46 \mathrm{~cd}$ & 15,2 & $2,28 \mathrm{c}$ & $2,62 \mathrm{e}$ & $1,95 \mathrm{ef}$ & 34,6 \\
\hline C. pyriformis & $70,5 \mathrm{a}$ & $71 \mathrm{ab}$ & $70 \mathrm{ab}$ & 1,4 & $1,13 \mathrm{~d}$ & $1,38 \mathrm{~cd}$ & $0,87 \mathrm{~d}$ & 59,4 & $2,27 \mathrm{c}$ & $2,29 \mathrm{ef}$ & $2,26 \mathrm{ef}$ & 1,3 \\
\hline A. excelsum & $73,5 \mathrm{a}$ & $81 \mathrm{a}$ & $66 \mathrm{~b}$ & 22,7 & $2,92 \mathrm{c}$ & $4,71 \mathrm{~b}$ & $1,13 \mathrm{~cd}$ & 318,2 & $3,57 \mathrm{~b}$ & $5,40 \mathrm{c}$ & $1,74 \mathrm{f}$ & 210,8 \\
\hline B. quinata & $74,0 \mathrm{a}$ & $74 \mathrm{ab}$ & $74 \mathrm{ab}$ & 0 & $5,71 \mathrm{a}$ & $5,65 \mathrm{a}$ & $5,77 \mathrm{a}$ & 0 & $7,06 \mathrm{a}$ & $6,73 \mathrm{~b}$ & $7,40 \mathrm{~b}$ & $-9,1$ \\
\hline \multicolumn{1}{|c|}{ Media } & 73,90 & $76,8 \mathrm{a}$ & $71,0 \mathrm{a}$ & 8,2 & 3,00 & $3,83 \mathrm{a}$ & $2,17 \mathrm{~b}$ & 76,5 & 4,38 & $5,23 \mathrm{a}$ & $3,53 \mathrm{~b}$ & 48,2 \\
\hline
\end{tabular}

Promedios con la misma letra no difieren estadísticamente, según la prueba de Duncan, comparación vertical entre especies y horizontal entre ambientes. PG: Porcentaje de germinación acumulado; IVG: Índice de velocidad germinativo; GDM: Germinación diaria media. Media: media general / especie; CAGER: cámara germinativa; CAMA: casa-malla; INC: incremento de CAGER sobre CAMA. 
A nivel de los dos ambientes germinativos, $C$. odorata y $B$. quinata registraron mayor valor promedio del IVG en CAGER (5,72 y 5,65 plántulas/día, respectivamente), mientras que C. pyriformis y S. parahybum registraron los valores más bajos con 1,38 y 1,69 plántulas/día, en su orden. En CAMA, el mayor IVG se presentó en $B$. quinata, con promedio de 5,77 plántulas/día, mientras que el valor más bajo del IVG, se observó en C. pyriformis, con 0,87 plántulas/día, respectivamente. Adicionalmente, los resultados muestran que $B$. quinata fue también la especie que registró la menor diferencia entre las dos condiciones ambientales de germinación, con un IVG promedio similar en CAGER y CAMA. Por el contrario, se observó en A. excelsum y C. odorata, diferencias de gran magnitud entre ambos ambientes en el IVG (318\% y $251 \%$, respectivamente), a favor de los resultados en CAGER. En promedio, se observó mayor expresión del IVG en condiciones de laboratorio en CAGER comparado con CAMA, con un valor promedio de $76,5 \%$. La explicación detallada para estos resultados, se presenta al final en forma conjunta para las especies y los parámetros de germinación, que mostraron un comportamiento similar en los dos ambientes evaluados.

Germinación diaria media (GDM). En la tabla 2, se observa que, en general, las especies $B$. quinata y $C$. odorata presentaron los mayores promedios de GDM, con 7,06\% y $6,70 \%$, respectivamente; estos valores superan a la media general (4,38\%), en 2,7 y 2,3 puntos, aproximadamente. Por su parte, C. pyriformis y S. parahybum mostraron los menores valores promedios de GDM, con 2,27\% y 2,28\% de las semillas germinadas por día. Estas diferencias se explican, posiblemente, por las características morfológicas y anatómicas propias de las semillas, lo cual, permite una absorción más rápida de agua y, por ende, se inicie y genere una germinación total más rápida de las semillas en $B$. quinata y $C$. odorata, que exhibieron los mayores valores promedios de GDM.

La especie que en CAGER mostró estadísticamente la mayor GDM fue $C$. odorata, con el 9,11\% de las semillas germinadas en promedio/día, mientras que el menor porcentaje correspondió a C. pyriformis y S. parahybum, con 2,29 y $2,62 \%$, respectivamente. En CAMA, la mayor GDM se presentó en $B$. quinata, con el 7,40\% de las semillas germinadas en promedio/día y los menores porcentajes, en A. excelsum, S. parahybum y C. pyriformis, con 1,74, 1,95 y $2,26 \%$, respectivamente. De igual manera, los datos señalan que $C$. pyriformis fue la especie más consistente y menos afectada por las condiciones de germinación, dado que su GDM exhibió promedios similares en CAGER y CAMA; situación opuesta se detectó en $A$. excelsum y C. odorata, en donde los dos ambientes de germinación presentaron diferencias en el GDM, del 210,8 y 112,2\%, respectivamente, a favor de las respuestas en CAGER. En promedio, las cinco especies presentaron un 48,3\% de mayor expresión de la GDM, en condiciones de CAGER, comparado con CAMA.

Valor pico de la germinación (VP). En la tabla 3, se detalla que la media general para el VP, entre las especies, osciló entre $2,96 \%$, para $C$. pyriformis y $15,33 \%$, para B. quinata; esta especie superó en 7,27 puntos a la media general para todo el estudio, que fue de $8,06 \%$. Las especies que en CAGER, bajo condiciones de laboratorio mostraron estadísticamente los valores de mayor magnitud del VP, fueron $C$. odorata y $B$. quinata, con un máximo de semillas germinadas/día de 16,10 y $15,33 \%$ respectivamente; los menores promedios se presentaron en $S$. parahybum y $C$. pyriformis, con 3,41 y $3,43 \%$, en su orden. En CAMA, el máximo porcentaje de semillas germinadas en un día, se presentó en $B$. quinata, con un valor de $15,33 \%$, mientras que el menor registro de VP de germinación se obtuvo en $C$. pyriformis, S. parahybum, A. excelsum y C. odorata, con promedios de 2,50, 3,19, 3,22 y 4,66\%, respectivamente. Los datos indican, además, que $B$. quinata fue también la especie más estable y menos afectada en este parámetro, por las condiciones de germinación, dado que su VP fue igual en CAGER y en CAMA, con 15,33\%, máximo de semillas germinadas en un día, durante la prueba. Caso contrario se observó en $A$. excelsum y $C$. odorata, en donde las dos condiciones de germinación representaron diferencias en el VP, del 316,1 y $245,9 \%$, respectivamente, a favor de los resultados en CAGER. En promedio, se observó mayor expresión del VP, en condiciones de CAGER, comparado con CAMA, con una diferencia del $78,7 \%$.

Valor de germinación (VG). La prueba de comparación de medias para este parámetro fisiológico de la germinación señala diferencias estadísticas entre las especies (E), ambientes germinativos (A) e interacción ExA (Tabla 3). En general, C. pyriformis y S. parahybum registraron los valores más bajos de VG, con 6,80 y 7,67 unidades en su orden, mientras que $B$. quinata y $C$. odorata registraron los mayores valores promedios de VG, con 109,71 y 83,61, respectivamente; estos valores representaron a la media general, en 2 veces, aproximadamente.

En los ambientes germinativos, C. odorata registró estadísticamente el mayor valor medio de VG, con 147,11 unidades en CAGER, mientras que el menor valor promedio se registró en C. pyriformis y S. parahybum, con 7,91 y 9,04 unidades, respectivamente. Bajo las condiciones de CAMA, el mayor VG se presentó en B. quinata, con 114,63 y los menores valores promedios se registraron en C. pyriformis, A. excelsum, S. parahybum, y C. odorata, con 5,68, 5,78, 6,30 y 20,11 unidades, respectivamente. Adicionalmente, los datos señalan que $B$. quinata fue la especie más consistente y menos afectada por las condiciones de germinación, dado que su VG exhibió promedios similares en CAGER y CAMA, 
Tabla 3. Promedios de valor pico de la germinación (VP) y valor germinativo (VG) de las semillas en cámara germinativa (CAGER) y casa-malla (CAMA), de cinco especies forestales nativas en Córdoba.

\begin{tabular}{|l|c|c|c|c|c|c|c|c|}
\hline \multirow{2}{*}{ Especies } & \multicolumn{4}{|c|}{ VP (\%) } & \multicolumn{4}{c|}{ VG (\#) } \\
\cline { 2 - 10 } & Media & CAGER & CAMA & INC (\%) & Media & CAGER & CAMA & INC (\%) \\
\hline C. odorata & $10,38 \mathrm{~b}$ & $16,10 \mathrm{a}$ & $4,66 \mathrm{c}$ & 245,9 & $83,61 \mathrm{a}$ & $147,11 \mathrm{a}$ & $20,11 \mathrm{~d}$ & 631,6 \\
\hline S. parahybum & $3,30 \mathrm{c}$ & $3,41 \mathrm{c}$ & $3,19 \mathrm{c}$ & 7,0 & $7,67 \mathrm{c}$ & $9,04 \mathrm{~d}$ & $6,30 \mathrm{~d}$ & 43,6 \\
\hline C. pyriformis & $2,96 \mathrm{c}$ & $3,43 \mathrm{c}$ & $2,50 \mathrm{c}$ & 37,3 & $6,80 \mathrm{c}$ & $7,91 \mathrm{~d}$ & $5,68 \mathrm{~d}$ & 39,3 \\
\hline A. excelsum & $8,31 \mathrm{~b}$ & $13,40 \mathrm{~b}$ & $3,22 \mathrm{c}$ & 316,1 & $39,39 \mathrm{~b}$ & $73,01 \mathrm{c}$ & $5,78 \mathrm{~d}$ & 1164,3 \\
\hline B. quinata & $15,33 \mathrm{a}$ & $15,33 \mathrm{ab}$ & $15,33 \mathrm{ab}$ & 0 & $109,71 \mathrm{a}$ & $104,78 \mathrm{~b}$ & $114,63 \mathrm{~b}$ & $-8,6$ \\
\hline \multicolumn{1}{|c|}{ Media } & 8,06 & $10,33 \mathrm{a}$ & $5,78 \mathrm{~b}$ & 78,7 & 49,43 & $68,37 \mathrm{a}$ & $30,50 \mathrm{~b}$ & 124,2 \\
\hline
\end{tabular}

Promedios con la misma letra no difieren estadísticamente, según la prueba de Duncan, comparación vertical entre especies y horizontal entre ambientes VP: Valor pico y VG: Valor germinativo. Media: media general / especie; CAGER: cámara germinativa; CAMA: casa-malla; INC: incremento de CAGER sobre CAMA.

lo cual, no representa diferencias importantes en los dos ambientes. Situación opuesta se detectó en A. excelsum y C. odorata, en donde las dos condiciones de germinación representaron diferencias aproximadas en el VG, del 1,164\% y $632 \%$, respectivamente, a favor de los resultados obtenidos en CAGER. En promedio, se observó, una vez más, mayor expresión del VG, en condiciones de CAGER, comparado con CAMA, lo que representó un incremento promedio del $124,2 \%$.

La mayor expresión promedio de los parámetros fisiológicos de la germinación de las semillas IVG, GDM, VP y VG de las cinco especies, en las pruebas realizadas en CAGER, se explica en función del mayor control y uniformidad en las condiciones de sustrato, humedad, oxígeno, temperatura y luz para la germinación de las semillas, que se ofrece en el ambiente de la cámara germinativa. En casa-malla, las condiciones son muy heterogéneas y cambiantes, ya que dependen de las variaciones de clima reinantes durante el periodo de realización de las pruebas de germinación, como lo reportan varios autores: Olmez et al. (2009), Gokturk et al. (2012), Correa et al. (2013), Armaki et al. (2013) y Armaki (2014).

B. quinata fue la especie más consistente y menos afectada por las dos condiciones de germinación, dado que sus parámetros germinativos PG, IVG, GDM, VP y VG presentaron promedios muy similares en CAGER y CAMA, hecho que se puede explicar por tener una semilla con testa porosa, permeable y ligera de peso, lo que le confiere, a su vez, rápida absorción de humedad, menos días a inicio de la germinación, menos días durante la germinación, menos días para completar su germinación y el mayor IVG (Tabla 2). Esto permite clasificar a B. quinata como una de las especies con mayor energía y vigor germinativo, dado que el IVG, GDM, VP y VG estiman la energía y vigor para germinar de las semillas (Maguire, 1962). Situación contraria se detectó en $A$. excelsum, que fue la especie más afectada por los dos ambientes de germinación, en razón a que mostró las mayores diferencias en los cinco parámetros germinativos. Este comportamiento opuesto en las dos especies, se puede explicar por las características propias de las semillas de $A$. excelsum, que resultaron más pesadas, con testa dura y semi-impermeable; esto, a su vez conlleva a mayor resistencia mecánica a la absorción de humedad, mayores días a inicio de la germinación, más días durante la germinación, mayores días para completar su germinación y valores intermedios en IVG, GDM, VP y VG (Tabla 2), en las dos condiciones de evaluación del estudio.

En $B$. quinata, el proceso de germinación es muy rápido; la semilla presenta alta energía y vigor germinativo, además se ve favorecida en la CAGER, por el mayor control y uniformidad en las condiciones de germinación comparado con la casa-malla, en donde los micro-ambientes, al ser de menor control, cambiantes y muy heterogéneos, afectan negativamente y en mayor grado la evaluación de los parámetros de la germinación a las especies, con las características de las semillas de $A$. excelsum, como lo han reportado varios autores (Olmez et al. 2009; Gokturk et al. 2012; Armaki et al. 2013; Armaki, 2014).

Otro factor a tener en cuenta, a nivel de especie o condiciones de evaluación, sobre todo en casa-malla, es que el estrés por sequía, temperaturas extremas, radiación solar, salinidad o acidez del sustrato, costras de tierra que cubren la semilla, así como los agentes patógenos o herbívoros, podrían afectar negativamente los parámetros de germinación y de crecimiento de las plántulas, lo que contribuye a que 
las semillas que emergen tarde tengan menor capacidad competitiva en vivero y campo, que las semillas que emergen temprano (Armaki, 2014; Zohra et al. 2014).

Las especies o lotes de semillas que presentan los mayores PG, IVG, VP, GDM y VG, en condiciones óptimas de germinación son más deseables y de mejor calidad fisiológica, ya que permiten obtener el mayor número de plántulas, más vigorosas y en el menor tiempo posible, por su mayor energía y vigor germinativo. Lo anterior es más valioso si se tiene en cuenta que en teoría, probablemente, sólo las semillas que germinan con rapidez y vigor en condiciones favorables de laboratorio, casa-malla o invernadero, serán capaces de producir plántulas vigorosas en condiciones de vivero o mayor sobrevivencia inicial en campo (Gokturk et al. 2012; Rivera-Martin et al. 2013).

Adicionalmente, en la realización de las pruebas en cámara de germinación en laboratorio es posible manipular los factores que limitan la germinación de las semillas, hasta encontrar las condiciones óptimas para cada especie, lo cual, permite maximizar su capacidad de germinación. La utilización de condiciones ideales estandarizadas en la cámara germinativa, garantiza que las diferencias entre los resultados se pueden adscribir a diferencias reales entre muestras de semillas y no a diferentes métodos de análisis. Los resultados obtenidos para un determinado lote de semillas, bajo condiciones similares en CAGER en un laboratorio, serán parecidos a los obtenidos en cualquier otra cámara germinativa; los resultados serán reproducibles (Olmez et al. 2009; Gokturk et al. 2012; Correa et al. 2013; Armaki et al. 2013; Armaki, 2014).

Elconocimiento delosparámetros fisiológicos delagerminación de las cinco especies nativas en estudio contribuye a conocer la cantidad y las características de plántulas útiles a obtener por determinada cantidad de semilla sembrada; además de coadyuvar a entender la dinámica de la regeneración y establecimiento natural por semillas de las especies en los bosques naturales o plantados, donde deben enfrentar una serie de condiciones adversas bióticas y abióticas, que pueden restringir el éxito de la germinación y el crecimiento inicial de las plántulas (Rivera-Martin et al. 2013).

Por otro lado, los resultados de viabilidad, a través de una prueba de tetrazolio, acompañados con una posterior prueba de germinación en cámara germinativa, permitirán conocer el potencial teórico real de la calidad fisiológica de las semillas, que son de gran valor en los procesos de conservación, almacenamiento, monitoreo, regeneración, certificación y comercialización de las semillas, para los trabajos exitosos en viveros, silvicultura, mejoramiento genético y conservación del germoplasma (Nascimento, 2013; Armaki et al. 2013; Lazarotto et al. 2013; Abbade \& Massanori, 2014; Deminicis et al. 2014; Armaki, 2014; Guimarães et al. 2015).

Hay que tener presente que los resultados obtenidos bajo condiciones ideales controladas en CAGER en laboratorio no son directamente aplicables en el vivero, donde sólo se puede ejercer un control limitado sobre las condiciones ambientales, aunque, en la mayoría de casos, los dos resultados están estrechamente relacionados (Gokturk et al. 2012; Armaki et al. 2013; Armaki, 2014). En esta forma, el viverista, gradualmente, estará en capacidad de pronosticar el desempeño en vivero, basado en la germinación de CAGER en laboratorio; además, cada productor debe aplicar su propio factor de corrección, derivado de la experiencia, para convertir el porcentaje germinativo de un lote, tal como viene determinado por los ensayos en laboratorio, en la germinación efectiva que obtendrá en el vivero (Gokturk et al. 2012; Armaki et al. 2013; Guimarães et al. 2015).

Se concluye, en este estudio, que la respuesta de las especies fue diferente en los dos ambientes germinativos, para el índice de velocidad de germinación, germinación diaria media, valor pico y valor de germinación. Cuando la infraestructura lo permita, se recomienda mejor utilizar cámaras germinativas en laboratorio frente a casa-malla, para estimar los parámetros fisiológicos de la germinación de las semillas forestales, debido al mayor control que se ejerce sobre los factores ambientales esenciales para la misma.

Conflicto de intereses: El manuscrito fue preparado y revisado con la participación de todos los autores, quienes declaramos que no existe conflicto de intereses que ponga en riesgo la validez de los resultados presentados. Financiación: Este estudio fue cofinanciado en la convocatoria interna de proyectos de investigación de 2011 de la Universidad de Córdoba, el cual, se ejecutó bajo la alianza estratégica Universidad de Córdoba y Cadena Forestal de Córdoba (FORCARIBE).

\section{BIBLIOGRAFÍA}

1. ABBADE, L.; MASSANORI, T. 2014. Teste de tetrazólio para avaliação da qualidade de sementes de Tabebuia roseoalba (Ridl,) Sandwith-Bignoniaceae, submetidas ao Armazenamento. Rev. Árvore (Brasil). 38(2):233-240.

2. ARMAKI, M.A. 2014. The effects of metabolism in response to water stress of three Poa species under germinator and greenhouse conditions. Int. J. Agr. Forestry Fisheries (USA). 2(2):22-28.

3. ARMAKI, M.A.; HASHEMI, M.; AZARNIVAND, H. 2013. Physiological and morphological responses of 
three Bromus species to drought stress at seedling stage and grown under germinator and greenhouse conditions. Afr. J. Plant Sci. (India). 7(5):155-161.

4. BARBOZA-NOGUEIRA, F.C.; LOBO-PINHEIRO, CH.; MEDEIROS-FILHO, S.; DA SILVA MATOS, D.M. 2014. Seed germination and seedling development of Anadenanthera colubrina in response to weight and temperature conditions. J. Plant Sci. (India). 2(1):37-42.

5. CAMPO-ARANA, R.; EZQUIVEL-URANGO, N.; ESPITIACAMACHO, M. 2014. Hongos asociados a la semilla de seis forestales nativos, cultivados en el departamento de Córdoba. Fitopat. Col. 38 (2):27-31.

6. CORREA, E.; ESPITIA, M.; ARAMÉNDIZ, H.; MURILLO, O.; PASTRANA, I. 2013. Variabilidad genética en semillas de árboles individuales de Tectona grandis L.f, en la conformación de lotes mezclados en Córdoba, Colombia. Rev. U.D.C.A Act. \& Div. Cient. 16(2):379-389.

7. CRUZ, C. 2014. Programa genes. Versão Windows. Aplicativo computacional em genética e estatística. Versión 2009.7.0. Universidade Federal de Viçosa. Disponible desde internet en: http:///www,ufv,br/dbg/ genes/genes,htm (con acceso: 05/04/2014).

8. CZABATOR, F. 1962. Germination value: An index combining speed and completeness of pine seed germination. Forest Science (USA). 8(4):386-396.

9. DEMINICIS, B.B.; RODRIGUES, P.D.R.; FARIA, B.P.; VIEIRA, H.D.; PANDOLFI-FILHO, H.D.; FREITAS, G.S. 2014. Tetrazolium test to evaluate Stizolobium aterrimum seeds quality. Am. J. Plant Sci. (USA). 5(1):148-152.

10. FERREIRA, C.D.; SOUTO, P.C.; LÚCIO, A.F.; SOUTO, J.S.; SOUZA, B.V. 2012. Avaliações biométricas e germinação de sementes de Coaçu (Triplaris surinamensis Cham). Rev. Bras. Tecn. Apli. Ciên. Agrá. 5(1):147-162.

11. GOKTURK, A.; OLMEZ, Z.; KARASAH, B.; SURAT, H. 2012. Effects of cold stratification and sulphuric acid pre-treatments on germination of pomegranate (Punica granatum L.) seeds in greenhouse and laboratory conditions. Scientific Res. Essays (Nigeria). 7(25):2225-2229.

12. GOLD, K.; LEÓN-LOBOS, P.; WAY, Y.M. 2004. Manual de recolección de semillas de plantas silvestres para conservación a largo plazo y restauración ecológica. Instituto de Investigaciones Agropecuarias, Centro Regional de Investigación Intihuasi, La Serena, Chile. Boletín INIA $N^{\circ}$ 110. 62p. Disponible desde Internet en: http://www.inia.cl/medios/biblioteca/boletines/ NR31275.pdf (con acceso: 08/09/2012).

13. GUIMARÃES, R.R.; LEÃO, L.; NERY, M.C.; DE SOUZA, A.R.; CRUZ, S.M.; DE RESENDE, P.C.A. 2015. Tetrazolium test in crambe seeds. Semina: Ciências Agrárias (Brasil). 36(4):2539-2544.

14. HUERTA-PANIAGUA, R.; RODRÍGUEZ-TREJO, D. 2011. Efecto del tamaño de semilla y la temperatura en la germinación de Quercus rugosa Née. Rev. Chapingo Serie Ciencias For. Ambiente (México). 17(2):179187.

15. LAZAROTTO, M.; BRIÃO, M.F.M.; BELTRAME, R.; DOS SANTOS, A.F.; MEZZOMO, R.; PIVETA, G.; BLUME, E. 2013. Qualidade fisiológica e tratamentos de sementes de Cedrela fissilis procedentes do sul do Brasil. Rev. Árvore. 37(2):201-210.

16. MAGUIRE, D. 1962. Speed of germination-aid. In: Selection and evaluation for seedling emergence and vigour. Crop Sci. (USA). 2(1):176-177.

17. MWASE, W.F.; MVULA, T. 2011. Effect of seed size and pre-treatment methods of Bauhinia thonningii Schum, on germination and seedling growth. Afr. J. Biotechnol. (Nigeria). 10(13):5143-5148.

18. NASCIMENTO, I. 2013. Determinação de metodologias para teste de germinação e vigor de sementes de quixabeira (Bumelia obtusifolia Roem et Schult. var. excelsa (DC) Mig.). Rev. Árvore. 37(4):701-706.

19. NIEMBRO, A.; RAMÍREZ-GARCÍA, E. 2006. Evaluación de la cantidad y calidad biológica de semillas de caoba (Swietenia macrophylla King- Meliaceae) procedentes de una plantación en el estado de Campeche-México. Foresta Veracruzana (México). 8(1):23-30.

20. OLMEZ, Z.; GOKTURK, A.; KARASAH, B.; YILMAZ, H. 2009. Effects of cold stratification, and sulphuric acid pretreatments on germination of three provenances of smoke-tree (Cotinus coggygria Scop.) seeds in greenhouse and laboratory conditions. Afr. J. Biotechnol. (Nigeria). 8(19):4964-4968.

21. PALENCIA, G.; MERCADO, T.; COMBATT, E. 2006. Estudio agroclimático del departamento de Córdoba. 
Facultad de Ciencias Agrícolas. Ed. UNICOR (Colombia). 126p.

22. RIVERA-MARTIN, L.E.; PEÑUELA-MORA, M.C.; JIMÉNEZ-ROJAS, E.M.; VARGAS-JARAMILLO, M. 2013. Ecología y silvicultura de especies útiles amazónicas: Abarco (Cariniana micrantha Ducke), Quinilla (Manilkara bidentata (A, DC,) A, Chev,) y violeta (Peltogyne paniculata Benth), U.N. de Colombia (Sede Amazonas - Colombia) - Inst. Amazónico de Investigaciones. IMANI. 180p.

23. SILVA, K.B.; ALVES, E.U.; MATOS, V.P.; BRUNO, R.L. 2012. Caracterização morfológica de frutos, sementes e fases da germinação de Pachira aquatica Aubl, (Bombacaceae). Rev. Ciênc. Agr. (Brasil). 33(3): 891-898.
24. WANG, J.H.; BASKIN, C.C.; CHEN, W.; DU, G.Z. 2010. Variation in seed germination between populations of five sub-alpine woody species from Eastern Qinghai-Tibet Plateau following dry storage at low temperatures. Ecol. Res. (Japan). 25(1):195-203.

25. ZOHRA, B.; ALI, M.; MOULAY, B. 2014. Germination tests of seeds of argan tree (Argania spinosa (L.) Skeels) of two sources (Tindouf and Mostaganem) in the semi-arid western Algerian. Afr. J. Plant Sci. (India). 8(6):260-270.

Recibido: Marzo 19 de 2016

Aceptado: Julio 14 de 2016

\section{Cómo citar:}

Espitia, M.; Cardona, C.; Araméndiz, H. 2016. Pruebas de germinación de semillas de forestales nativos de Cordoba, Colombia, en laboratorio y casa-malla. Rev. U.D.C.A Act. \& Div. Cient. 19(2): 307-315. 